\section{BRAZIULIAN JOURNAL}

OF MEDICAL AND BIOLOGICAL RESHARCH

www.bjournal.com.br
ISSN 0100-879X

Volume 43 (11) 1010-1134 November 2010

BIOMEDICAL SCIENCES

AND

CLINICAL INVESTIGATION

Braz J Med Biol Res, November 2010, Volume 43(11)1076-1083

doi: 10.1590/S0100-879X2010007500112

Transplantation of muscle-derived stem cells plus biodegradable fibrin glue restores the urethral sphincter in a pudendal nerve-transected rat model

Y. Xu, Y.F. Song and Z.X. Lin

The Brazilian Journal of Medical and Biological Research is partially financed by

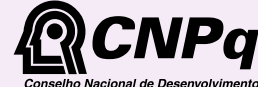

Ministério Conselho Nacional de e d
Cientifico e Tecnológico

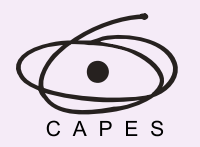

Ministério da Educação

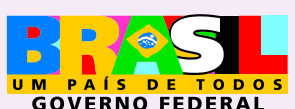

GOVERNO FEDERAL
TFAPESP

Institutional S ponsors

Hotsite of proteomics metabolomics developped by:

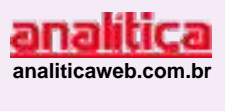




\title{
Transplantation of muscle-derived stem cells plus biodegradable fibrin glue restores the urethral sphincter in a pudendal nerve-transected rat model
}

\author{
Y. Xu1,2, Y.F. Song ${ }^{1,2}$ and Z.X. Lin $^{3}$ \\ ${ }^{1}$ Fuzong Clinical College, Fujian Medical University, Fuzhou, Fujian, China \\ ${ }^{2}$ Department of Obstetrics and Gynecology, Fuzhou General Hospital, Fuzhou, Fujian, China \\ ${ }^{3}$ Fujian Institute of Hematopathy, Fujian Medical University Union Hospital, Fuzhou, Fujian, China
}

\begin{abstract}
We investigated whether fibrin glue (FG) could promote urethral sphincter restoration in muscle-derived stem cell (MDSC)-based injection therapies in a pudendal nerve-transected (PNT) rat, which was used as a stress urinary incontinence (SUI) model. MDSCs were purified from the gastrocnemius muscles of 4-week-old inbred female SPF Wistar rats and labeled with green fluorescent protein. Animals were divided into five groups $(N=15)$ : sham $(S)$, PNT (D), PNT+FG injection (F), PNT+MDSC injection (M), and PNT+MDSC+FG injection (FM). Each group was subdivided into 1-and 4-week groups. One and 4 weeks after injection into the proximal urethra, leak point pressure (LPP) was measured to assess urethral resistance function. Histology and immunohistochemistry were performed 4 weeks after injection. LPP was increased significantly in FM and M animals after implantation compared to group $D(P<0.01)$, but was not different from group $S$. LPP was slightly higher in the FM group than in the $\mathrm{M}$ group but there was no significant difference between them at different times. Histological and immunohistochemical examination demonstrated increased numbers of surviving MDSCs (109 \pm 19 vs $82 \pm 11 / \mathrm{hpf}, \mathrm{P}=0.026)$, increased muscle/ collagen ratio $(0.40 \pm 0.02$ vs $0.34 \pm 0.02, P=0.044)$, as well as increased microvessel density $(16.9 \pm 0.6 \mathrm{vs} 14.1 \pm 0.4 / \mathrm{hpf}$, $\mathrm{P}=0.001$ ) at the injection sites in FM compared to $\mathrm{M}$ animals. Fibrin glue may potentially improve the action of transplanted MDSCs to restore the histology and function of the urethral sphincter in a SUI rat model. Injection of MDSCs with fibrin glue may provide a novel cellular therapy method for SUI.
\end{abstract}

Key words: Muscle-derived stem cells; Fibrin glue; Urinary incontinence; Cellular therapy; Pudendal nerve transection

\section{Introduction}

Stress urinary incontinence (SUI) is defined as involuntary leakage of urine on effort or exertion or during sneezing or coughing. It is a widespread health problem that adversely affects the quality of life of women $(1,2)$. Its etiology is multifactorial, including functional impairment of muscle and associated nerves that may occur as a result of advanced age, hormonal status, or pelvic floor damage at childbirth. Urethral injection therapy, such as bovine glutaraldehyde cross-linked collagen injection, is widely accepted as a less invasive SUI treatment modality with a relatively low risk of adverse events as well as good short-term results (3). However, the efficacy of such treatment declines with time, and repeated injections are required (4). Currently, cell-based injection therapies are being investigated as alternatives. Autologous adult stem cell therapy recently demonstrated the potential for regenerative repair of the deficient urethra for the treatment of SUI (5). Among adult stem cells, muscle-derived stem cells (MDSCs) possess several characteristics that are ideally suited for cellular transplantation. MDSC therapy appears to be effective in the regeneration of urethral sphincter in SUI animals and patients (6-8). However, the current transplantation technique involves the injection of cells suspended in saline, cell culture medium, or bovine serum albumin (BSA), and is plagued by limited cell retention and transplant survival $(9,10)$. Another main barrier to muscle tissue engineering

Correspondence: Y.F. Song, Fuzong Clinical College of Fujian Medical University, Fuzhou 350025, Fujian, China/Department of Obstetrics and Gynecology, Fuzhou General Hospital, Fuzhou 350025, Fujian, China. Fax: 0591-2493-7024. E-mail: syanfeng@yahoo.com

Received May 6, 2010. Accepted September 27, 2010. Available online October 15, 2010. Published November $12,2010$. 
for clinical therapy is the lack of an adequate vascular supply for larger volume implants (11). Cells at the center of an injected cell mass depend on an extensive blood vessel network, which supplies nutrients and oxygen while removing waste products (12). Many different strategies are currently being pursued to promote cell survival and retention. Tissue engineering approaches aim to repair lost or damaged tissue through the use of cell transplantation and biomaterial scaffolds, which provide a microporous framework and are etched with 'mini-networks', imitating vascular systems, that promote cell survival. To be clinically useful as cell transplant vehicles, such scaffolds need to be biocompatible without toxic side effects and clinical grade components need to be used to attach the cells to the scaffold. A liquid support matrix that polymerizes to a gel would be shaped more easily. In addition, an injection would be much less invasive than open implantation.

Fibrin glue (FG), a composite of fibrinogen and thrombin, is a physiologically relevant matrix whose principal component, fibrin, has a fundamental role in the process of blood clotting and wound healing. Thrombin is an endoprotease that naturally functions as a blood-clotting factor to convert fibrinogen to fibrin. Within seconds after fibrinogen and thrombin are mixed together, they form a gel-like component $(13,14)$. FG has been widely used to decrease intraoperative bleeding and to promote homeostasis. Fibrin is a natural polymer known to support wound healing by inducing angiogenesis and promoting cell attachment and proliferation, and thus may provide a more conducive environment for accelerated tissue regeneration $(15,16)$. While FG has been applied as a scaffold in tissue engineering $(17,18)$, it is not known if it could promote effective cellbased injection therapies in SUI.

In the present study, we explored the use of biodegradable FG scaffolds as an appropriate carrier to which primary rat MDSCs could be attached, and their use in vivo to support transplanted cells. Our model was designed to predict the potential of this technique for future clinical applications.

\section{Material and Methods}

\section{Animals and experimental design}

All experiments, including MDSC isolation and the denervation model, were performed on 75 normal inbred virgin female SPF Wistar rats (4 weeks old) weighing 80-100 $\mathrm{g}$. The rats were randomly divided into five groups: sham (S), pudendal nerve-transected (PNT, D), PNT+FG injection (F), PNT+MDSC injection (M), and PNT+MDSC+FG injection (FM). Groups were then subdivided into 1- $(\mathrm{N}=$ $5)$ and 4-week $(N=10)$ groups. The experimental protocol was approved by the Fuzhou General Hospital Institutional Animal Care and Use Committee.

Denervation of the bilateral pudendal nerve

Groups D, M, F, and FM underwent bilateral PNT (19).
Group S was the sham operation control. The rats were given chloral hydrate anesthesia (400 mg/kg, ip), and a dorsal midline incision in the skin and bilateral dorsal incisions in the muscle were performed over the ischiorectal fossa. Under an operating microscope, the sciatic nerve on each side was identified and a 2-mm segment distal to its origin from the vertebral column but proximal to the branch of the pudendal nerve was transected. The muscle and skin incisions were closed separately with 3-0 vicryl sutures. After the operation, animals were given a cefazolin sodium injection (100 mg/kg, ip) once a day for 3 days.

\section{MDSC isolation and purification}

MDSCs were isolated and purified from the hind gastrocnemius of 4-week-old normal inbred virgin female SPF Wistar rats via a previously described modified preplate technique (20). According to the results of previous studies on murine cells, early plates (preplates 1-2) contain a majority of fibroblasts, and late plates (preplates 5-6) are highly enriched with myogenic cells $(20,21)$. The cells used in the present experiment were taken from the last preplate, preplate 6, which contains MDSCs.

\section{Fibrin glue}

The FG used in this study is commercially available (BD Biosciences, USA). The two-component system remains liquid for several seconds before solidifying into a semirigid gel matrix. The first component consists of concentrated fibrinogen and aprotinin, a fibrinolysis inhibitor. The second component is a mixture of thrombin and $\mathrm{CaCl}_{2}$. It was delivered through the supplied applicator, which held the two components in separate syringes and provided simultaneous mixing and delivery. The ratio of fibrinogen to thrombin components was $1: 1(\mathrm{w} / \mathrm{w})$.

\section{MDSC labeling and injection surgeries}

To facilitate the identification of the cells after their transplantation, the MDSCs from the third passage were infected with lentivirus encoding green fluorescent protein (PGC-FU-GFP, ShangHai GeneChem Co., China) for $8 \mathrm{~h}$ in the presence of $8 \mu \mathrm{g} / \mathrm{mL}$ Polybrene at 20 multiplicities of infection. The transducing efficiency was up to $95 \%$. The transduced cells were expanded for 2 weeks before implantation. The injection surgeries were performed 2 weeks after bilateral PNT. A low midline incision was made to expose the urethra and different media were injected into the proximal urethra using a $100-\mu \mathrm{L}$ Hamilton syringe, with microscopic guidance on each side of the urethra. Five experimental groups of female rats were established. The cell injection group (M) was injected with $50 \mu \mathrm{L}$ MDSCs suspended in PBS ( $1 \times 10^{6}$ cells $\left./ 50 \mu \mathrm{L}\right)$; the FG group (F) was injected with $50 \mu \mathrm{L}$ of the mixed $F G$ via the Baxter supplied Duploject applicator; the FG and cell injection group (FM) was injected with $25 \mu \mathrm{L}$ MDSCs suspended in the thrombin $\left(1 \times 10^{6}\right.$ cells $\left./ 25 \mu \mathrm{L}\right)$ component with 25 
$\mu \mathrm{L}$ fibrinogen component. The $\mathrm{S}$ group was used as the positive control, which received no injection and the PNT group (D) was used as the negative control, which was injected with $50 \mu \mathrm{L}$ PBS.

\section{Leak point pressure test}

Leak point pressure (LPP) was determined in all animals at 1 and 4 weeks after injection as described by Damaser et al. (22). Briefly, after anesthesia with chloral hydrate $(400 \mathrm{mg} / \mathrm{kg}, \mathrm{ip})$, an abdominal midline incision of $0.5 \mathrm{~cm}$ was performed cephalad to the urethral meatus. A circular purse-string suture (6-0 chromic gut) was placed in the anterior vesical wall and a puncture incision was then made in the center of the purse-string stitch. A suprapubic catheter (PE-50 tubing) was inserted through this incision and secured with the suture. The catheter was subcutaneously tunneled to exit the skin at the level of the neck and the end was ligated. The bladder catheter was then connected to both a flow pump (P-600, Atom Medical Corp., Japan) and a pressure transducer via a 3-way stopcock. The transducer, connected to an amplifier, polygraph and computer, digitized the pressure data (UDS64-111, LABORIE Durado Urodynamic System, USA). The rat was placed in the supine position and underwent a 30 -min accommodation period of filling $(5 \mathrm{~mL} / \mathrm{h})$ and voiding. The bladder was then palpated, submitted to the Crede maneuver for emptying, and filled with saline to approximately half of its capacity. While bladder pressure was recorded and digitized, gentle pressure was applied externally over the bladder (a gentle Crede maneuver) to slowly increase pressure until the rat leaked saline through the urethra. At the first indication of leakage, the externally applied abdominal pressure was rapidly removed. The peak bladder pressure was taken as the LPP. The test was repeated at least three times to produce a mean LPP value for each rat.

\section{Histology}

After LPP testing at 4 weeks after injection, all animals were sacrificed. The proximal urethra was removed, and five specimens in each group were fixed in 10\% phosphatebuffered formalin, embedded in paraffin and sectioned into 5- $\mu \mathrm{m}$ thick slices. Paraffin sections were stained with Masson trichrome according to the manufacturer protocol (Sigma-Aldrich, USA). Images from the entire sections were acquired using a digital camera system (Imaging Micropublisher 5.0 PTV). To prevent variations in staining, all samples were stained simultaneously using this procedure. In the images, cells in blood vessels and the smooth muscle layer, as well as the rhabdosphincter layer in the sections, stained red while collagens stained blue. Image analysis was done as described above and quantified using the Image-Pro Plus 6.0 image software. The software can automatically distinguish regions stained with different colors and accurately measure the areas of muscle and collagen to yield a muscle/collagen ratio. Another five specimens from groups $\mathrm{FM}$ and $\mathrm{M}$ were fresh frozen in Tissue Tek O.C.T. freezing medium (Sakura, USA) at $-80^{\circ} \mathrm{C}$ and stored until they were processed on a cryostat. They were sectioned into 8- $\mu \mathrm{m}$ thick slices, and implanted cells were identified with GFP while the nuclei were stained with 4,6-diamidino2-phenylindole (DAPI, $1 \mu \mathrm{g} / \mathrm{mL}$; Roche, USA). For image analysis, five randomly selected fields per tissue per animal in the FM and M groups were photographed with an Confocal Laser Microscope (LCSM510, Zeiss, Germany) and the GFP-MDSCs were counted using the Image-Pro Plus image software.

\section{Histochemical staining for factor VIII-related antigen and microvessel density count}

For detailed immunohistochemical analysis of the repaired tissue, paraffin sections were probed with polyclonal rabbit anti-factor VIII-related antigen (FVIII-RAg, F8, Zymed, USA). Briefly, all sections were deparaffinized in xylene and treated with $3 \%$ hydrogen peroxide in methanol for $10 \mathrm{~min}$ to block endogenous peroxidase activity. Enzyme pretreatment for antigen retrieval was necessary. Nonspecific binding of primary antibodies was reduced by blocking with normal horse serum for $10 \mathrm{~min}$. Sections were then incubated in a humidified chamber at $4^{\circ} \mathrm{C}$ with the primary antibody (1:100) for $24 \mathrm{~h}$, followed by the second antibody using the MaxVision ${ }^{\mathrm{TM}}$ HRP-Polymer anti-Rabbit IHC Kit, (Maxim Co., China). Control sections omitted the primary antibody and were treated with PBS alone. The sections were stained with 3,3-diaminobenzidine tetrachloride solution (DAB) and counterstained with hematoxylin. The endothelial cells in blood vessels that stained brownish red were FVIII-R Ag+ cells. A microvessel was defined as any distinct FVIII-R $\mathrm{Ag}+\mathrm{cell}$ or cell cluster, with no requirement for a vessel lumen. The number of microvessels was counted in 10 random fields (magnification: 20X). The average of the 10 high power fields (hpf) was calculated and the microvessel density was defined as microvessel/hpf using an Olympus CX40 microscope (Tokyo, Japan).

\section{Statistical analysis}

Data are reported as means \pm SD. The software SPSS 11.0 for Windows (SPSS Inc., USA) was used for analysis. Comparison of continuous variables among the five groups was performed by one-way analysis of variance (ANOVA) for within time-point analyses. The LSD method was used to specify differences among groups. Paired $t$-tests were used for comparison between data at 1 and 4 weeks. A $P$ value less than 0.05 was considered to be statistically significant.

\section{Results}

\section{LPP testing}

The LPPS of the FM, M, F, D, and S groups at 1 and 4 weeks after injection are shown in Figure 1. Post hoc LSD 
analysis indicated that the LPP of group D was lower than that of other groups at each time $(P<0.01)$. The LPPs of group FM and $M$ were significantly higher than that of group $D(P<0.01)$ but there was no difference compared with group $S$ at either time. The LPP of group FM was slightly higher than that of group M, although the difference was not significant at either time. The LPP of group F was significantly higher than that of group $D(P=0.02)$ and significantly lower than that of group $S(P=0.025)$ at 1 week. At 4 weeks the LPP of group $F$ was significantly lower than that of groups $F M, M$ and $S(P=0.04, P=0.09$ and $P=0.01$, respectively $)$ but there was no difference compared to group $D(P=$ $0.276)$. There was a sharp decrease of the LPP in group $F$ from 1 to 4 weeks $(P=0.002)$, but no significant difference between 1 and 4 weeks in the other groups.

\section{Histology}

The implanted GFP-MDSCs were detected in the muscle layer of the proximal urethra at 4 weeks after the injection in both groups FM and M. The GFP-MDSCs implanted in

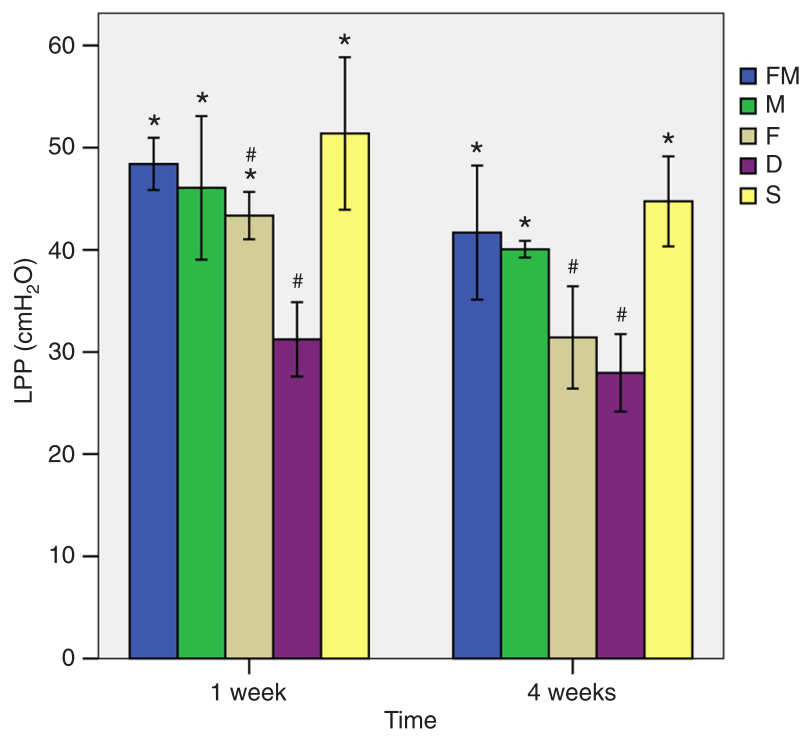

Figure 1. Leak point pressure (LPP) analysis 1 week and 4 weeks after injection. The LPP of the PNT+MDSC plus fibrin glue (FG) injection group (FM) and the PNT+MDSC injection group (M) was significantly higher than that of the PNT group (D) at 1 and 4 weeks $(P<0.01)$. There was no difference in LPP in either group compared with that of the sham group (S) at either time. The LPP of group FM was slightly higher than that of group M but there was no significant difference between the two groups at either time. The LPP of the PNT+FG injection group $(F)$ was significantly higher than that of group $D(P=0.002)$ and significantly lower than that of group $S(P=0.025)$ at 1 week, but there was no difference compared to that of group $D$ at 4 weeks. Data are reported as means $\pm S D$ ( $N=5$ for each group). $P N T=$ pudendal nerve transected; MDSC $=$ muscle-derived stem cells. ${ }^{*} \mathrm{P}<$ 0.05 compared with group $\mathrm{D}$; ${ }^{\mathrm{P}}<0.05$ compared with group $\mathrm{S}$ (ANOVA + LSD test). combination with FG seem to localize in the host tissue and fuse to form post-mitotic multinucleated myofibers, since the DAPI-stained nuclei showed varied sizes on the implanted side. There was an increase of surviving GFP-MDSCs in situ in group $F M$ compared to group $M(P<0.05$; Figure $2 A, B)$. Histological examination of the cross-section of the 4-week proximal urethra was performed on each section after Masson trichrome staining. In the normal female rat external urethral sphincter, the circular skeletal muscle fibers, a layer of striated muscle fibers that encircles the smooth muscle layers, and the inner longitudinal as well as middle circular layers were all stained red (Figure 3A). The
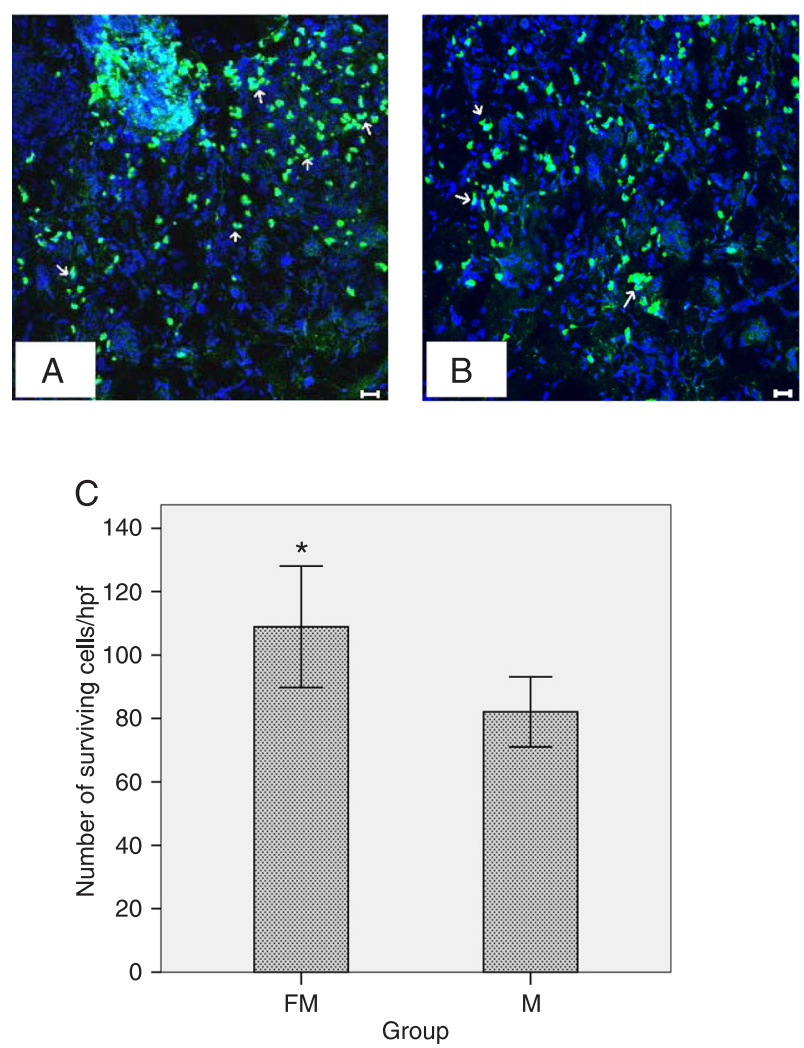

Figure 2. The implanted GFP-MDSCs were detected in the muscle layer of the proximal urethra 4 weeks after injection in both the PNT+MDSC plus fibrin glue (FG) injection group (FM) and the PNT+MDSC injection group (M). A, A greater number of surviving transplanted GFP-MDSCs localized at the injected sites and some of them had fused to each other to form post-mitotic multinucleated myofibers when rats were transplanted with MDSCs in combination with fibrin glue (white arrows). $B$, There was a smaller number of surviving transplanted GFP-MDSCs at the injected sites when rats were injected with MDSCs only. Original magnification 200X. Scale bar $=20 \mu \mathrm{m}$. C, There was an increase of surviving GFP-MDSCs in situ in group FM compared to group $M\left({ }^{*} \mathrm{P}<0.05, t\right.$-test). Data are reported as means $\pm S D$ $(\mathrm{N}=5$ for each group). GFP = green fluorescent protein; MDSC = muscle-derived stem cells; PNT = pudendal nerve transected; hpf $=$ high power field. 
denervated proximal urethral sphincter showed atrophic and thin skeletal and smooth muscle fibers (Figure 3B). The denervated proximal urethral sphincter injected with MDSC plus FG displayed an increased thickness of circular skeletal muscle at the injection sites (Figure 3C). The injection of MDSCs also displayed an increased thickness of muscle mass and the orientation of the new muscle fibers was variable (Figure $3 D$ ). The injection of FG led to an increased vessel density but few new muscle fibers could be found at the injection sites (Figure 3E). The muscle/ collagen ratios of the $\mathrm{FM}$ and $\mathrm{M}$ groups were significantly higher than in group $D(P<0.01)$ but lower than in group $S$ $(P<0.01)$. In group $F M$, the ratio was higher than in group $M(P=0.044)$. In addition, there was no difference between group $\mathrm{F}$ and group $\mathrm{D}$.

\section{Effects of MDSC plus fibrin glue transplantation on microvessel density}

The microvessel density of the different groups observed four weeks after injection is shown in Figure 4G. The microvessel density of the FM group was significantly higher than in the other groups $(P<0.01)$. After the injection of MDSCs plus FG, a large number of FVIII-R Ag-labeled microvessels were found within the striated muscle layer (Figure 4B). There was a moderate increase in microvessel density in the S, M and F groups (Figure 4A,C,D) compared with the $D$ group (Figure $4 \mathrm{E} ; \mathrm{P}<0.01$ ). There was no significant difference in microvessel density between the $M$ group and the $\mathrm{F}$ group.

\section{Discussion}

Several new materials are being investigated for injection therapy of SUI. Among these, MDSCs are especially promising because the cells are muscle progenitor cells that have shown the capacity to differentiate into bone, cartilage, nerve, and endothelium $(20,21)$. MDSCs have the potential to improve sphincter function by remodeling the damaged urethra, as well as acting to generate tissues with bulking properties $(23,24)$. However, MDSC injection therapy has certain disadvantages including migration and absorption, as is the case for other commercial injection agents. This calls for the development of novel strategies, like the tissue-engineering approach, to improve the retention and survival of the transplanted stem cells.

Clinically, LPP determination is currently used to diagnose SUI during a urodynamics exam. LPP by the Crede method represents the active component of the continence mechanism of the urethral sphincter in SUI rat models. Thus, it probably better reflects the state of urethral resistance function and has been used extensively in rat model studies (25-27). In the present study, we determined whether FG can improve the effect of MDSCs on urethral function in the denervated urethral sphincter of rats by detecting LPP 1 and 4 weeks after treatment. The results showed that the urethral function in the MDSC+FG injection group and the MDSC injection group was significantly improved at both times although there were no significant differences between groups. When FG was injected alone, LPP was increased slightly at 1 week but was decreased at 4 weeks. The reduction in volume of the FG, which acted as

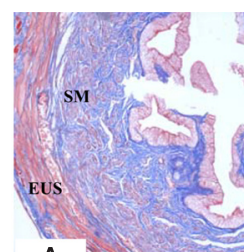

A
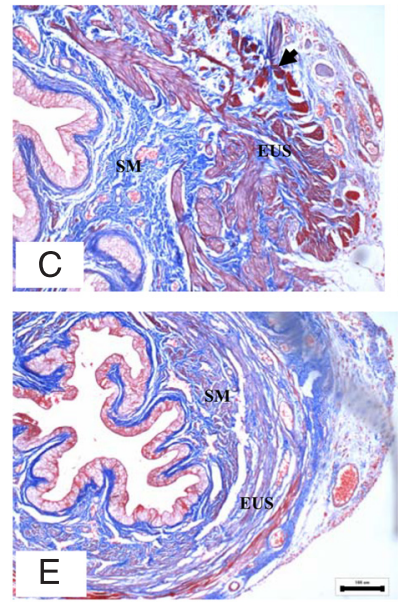
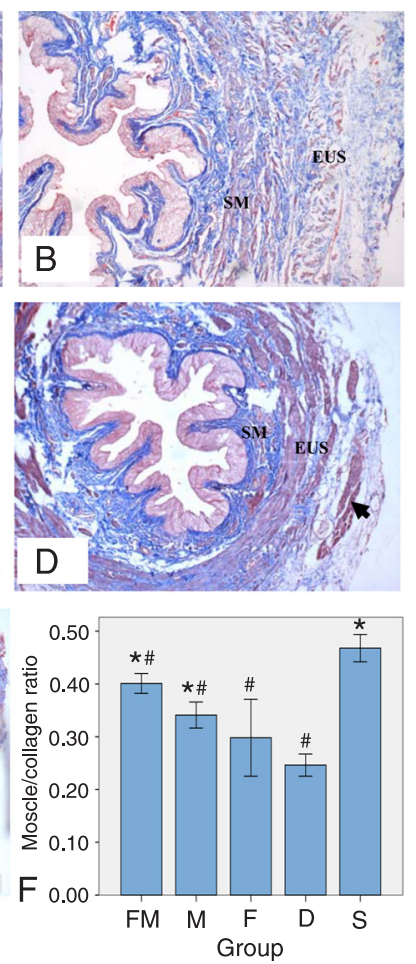

Figure 3. Masson trichrome staining of a female rat urethral sphincter 4 weeks after injection. $A$, Normal anatomic structure of the urethral sphincter from a sham-operated control female rat (group S). B, Following denervation (group D), the circular skeletal muscle and smooth muscle layer were thin and atrophic. $C$, The denervated proximal urethral sphincter of an animal injected with MDSCs plus fibrin glue (group FM) displayed an increased thickness of circular skeletal muscle and more new muscle fibers (black arrow) on the external urethral sphincter layer. D, MDSCs injected into a denervated proximal urethral sphincter (group M) led to increased skeletal muscle masses with variable fiber orientation in the external urethral sphincter layer. $E$, The denervated proximal urethral sphincter injected with fibrin glue alone (group F) displayed an increased vessel density but few new muscle fibers could be seen at the injection site. $F$, The muscle/collagen ratios were the highest in group $S$. They were significantly higher in groups $F M$ and $M$ than in group $D(P<0.01)$, but lower than in group $S(P<0.01)$. The muscle/collagen ratio of group $F M$ was higher than that of group $M(P=0.044)$. There was no difference between group $F$ and group $D$. ${ }^{*} P<0.01$ compared with $D$ group. ${ }^{\# P}<0.01$ compared with $S$ group (ANOVA + LSD test). Data are reported as means $\pm S D(N=5$ for each group). MDSCs = muscle-derived stem cells; EUS = external urethral sphincter; $\mathrm{SM}=$ smooth muscle. Original magnification 100X. Black arrows denote new muscle fibers. Scale bar $=100 \mu \mathrm{m}$. 

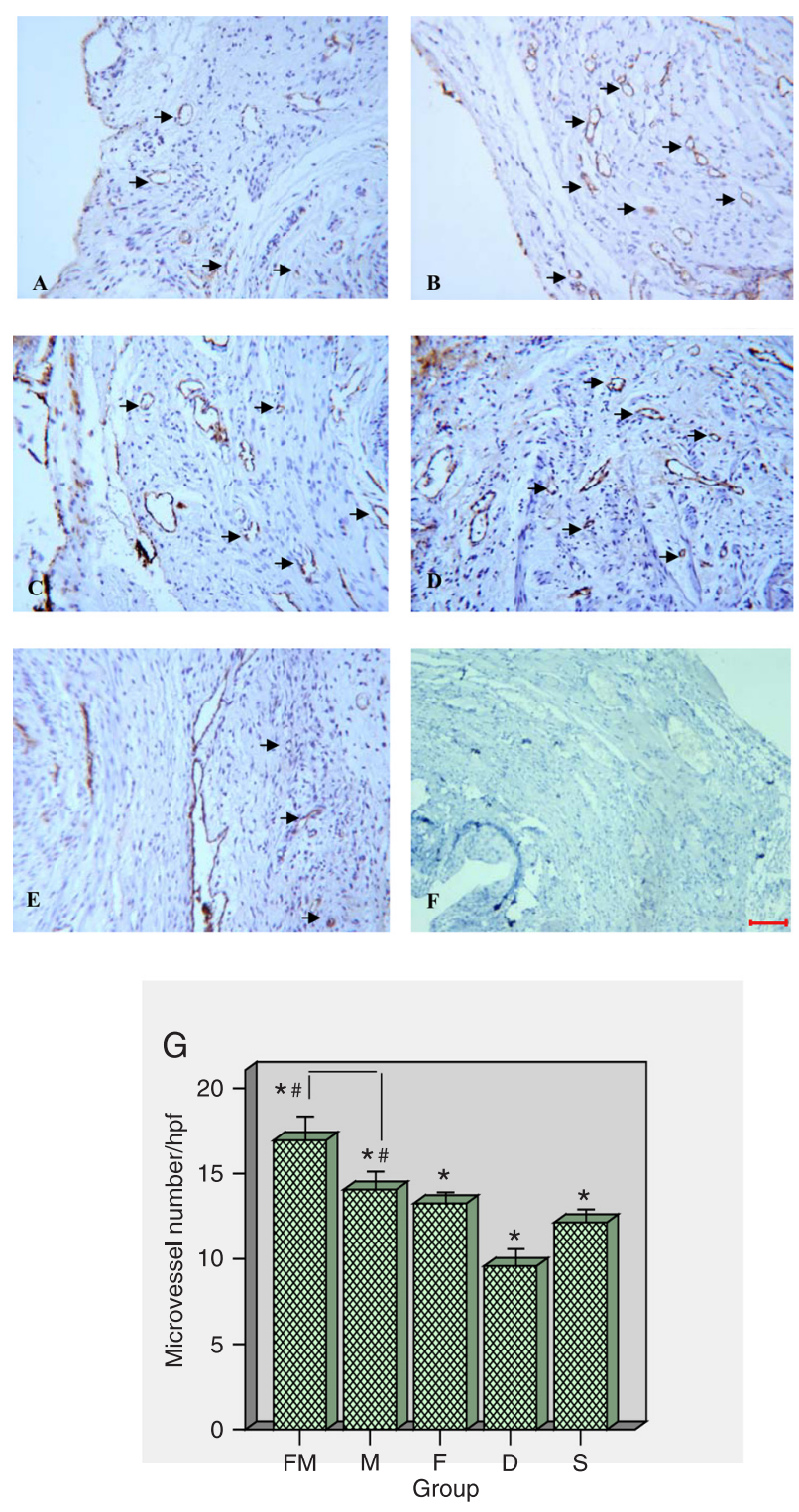

Figure 4. Histochemical analysis of the expression of factor VIIIrelated (FVIII-R) antigen and neovascularization 4 weeks after injection. Panels A-E show the capillaries with FVIII-R Ag+ cells (black arrows) compared to the negative control (PNT group, pudendal nerve transected; Panel F). After the injection of musclederived stem cells (MDSC) plus fibrin glue (FG), a large number of FVIII-R Ag-labeled microvessels were found within the striated muscle layer (Panel B). Microvessel density was highest in the PNT+MDSC plus FG injection (FM) group (Panel G). There was a moderate increase in microvessel density in the sham (S), PNT+MDSC injection (M) and PNT+FG injection (F) groups (Panels A,C,D) compared to the PNT (D) group (Panel E). *P < 0.01 compared with the control group $D$. ${ }^{\#} P<0.01$ compared with group $S$ (ANOVA + LSD test). Data are reported as means \pm SD $(\mathrm{N}=5$ for each group). Original magnification 200X. Scale bar $=$ $100 \mu \mathrm{m} . \mathrm{hpf}=$ high power field. a bulking agent, was responsible for the decline of LPP as it was gradually absorbed. These results demonstrate that the function of urethral sphincters was improved by the injection of MDSCs with or without FG while the injection of FG alone led to a temporary improvement. The lack of difference in LPP between the MDSC+FG injection group and the MDSC injection group may be attributed to the following reasons. First, in vivo, plasminogen and matrix metalloproteinases may be secreted from the encapsulated cells inside the FG, which contribute to its rapid degradation. Therefore, FG acting as a bulking agent may improve LPP temporarily. Second, it remains to be determined if FG can improve the differentiation of injected MDSCs into reinnervated myofibers for urethral sphincter contraction. Third, the lack of significance could simply be a type II error, with only 5 rats in each group, although the trends were actually quite strong.

Our results also indicate that survival of transplanted cells at the injection site 4 weeks after transplantation was enhanced by injection of FG. The histological and immunohistochemical analysis also showed an obvious increase of the thickness of muscle mass and of the muscle/collagen ratio, as well as a higher neovasculature density in the MDSC+FG injection group. These data indicate that FG might promote the differentiation of MDSCs toward a myogenic and endothelial lineage. In addition, fibrin-stabilizing factor XIII contained in FG favors the migration of undifferentiated stem cells on the highly cross-linked structure of the glue and enhances cell proliferation. FG also contains arginine-glycine-asparagine motifs and binds to integrins, which facilitate cell adherence and proliferation.

In the present study, improved LPPs after injection of MDSCs suggest that MDSCs could induce the functional reinnervation of regenerated muscles. The feasibility of this concept was first demonstrated by Chermansky et al. (28) who showed that the striated muscle layer of the MDSCinjected urethra was contiguous with an increase in nervous tissue when compared with those of the cauterized urethra injected with only saline solution in a SUI rat model. Furuta et al. (29) further suggested that the physiological effects of urethral sphincters were mediated not only by MDSC bulking but also by autonomic nerves innervating the urethral sphincters. MDSCs implanted into the proximal urethra might enhance a1-ARs sensitivity by differentiating into sympathetic neuronal cells or urethral smooth muscle cells to restore the deficient middle urethral function induced by transection of pudendal nerves. The transplanted MDSCs may also release NGF to promote axonal regeneration and functional recovery after nerve injury (30). However, it is likely that transplanted MDSCs may promote both the muscle and integrated nerve regenerative response of donor transplanted and host cells and that FG could promote such regenerative response.

FG in combination with an appropriate cell source has been used in a variety of tissue engineering applications, 
including maxillofacial bone, periodontal bone, bone, ear cartilage, cartilage, cornea, heart, blood vessel, tendon, and ligament regeneration (31). It has proved to be a potentially suitable biological vehicle for cell transplantation due to its injectability, biocompatibility, biodegradability, and binding capacity to cells. Unlike xenogenic gelatin and collagen, which may induce inflammatory responses, FG can avoid the potential risk of a foreign body reaction because it is a naturally occurring molecule that can be produced from proteins in the patient's own blood (32). Another advantage of using FG as a matrix is that it can be injected as a liquid and it will gel in situ. Injection of the fibrin/cell material leads to the formation of tissue that is histologically more mature. Our next step is to observe the therapeutic efficacy of MDSCs combined with FG injection in a rat model of SUI over a longer period of time.

The present short-term observation indicated that FG might potentially synergize with transplanted MDSCs to restore the histology and function of the urethral sphincter and promote the efficacy of cell-based injection therapies in a rat model of SUI. Thus, injection of MDSCs plus FG may provide a novel cellular therapy method for SUI.

\section{Acknowledgments}

Research supported by grants from the Key Science and Technology Planning Project of Fujian Provincial, China (grant\#2009I0022), National Natural Science Foundation of China (grant \#81070473) and Medical Science and Technology Foundation of Military Region (grant \#08MA099).

\section{References}

1. Abrams P, Cardozo L, Fall M, Griffiths D, Rosier P, Ulmsten $U$, et al. The standardisation of terminology in lower urinary tract function: report from the standardisation sub-committee of the International Continence Society. Urology 2003; 61: 37-49.

2. Hampel C, Wienhold D, Benken N, Eggersmann C, Thuroff JW. Prevalence and natural history of female incontinence. Eur Urol 1997; 32 (Suppl 2): 3-12.

3. Chapple CR, Wein AJ, Brubaker L, Dmochowski R, Pons $\mathrm{ME}$, Haab F, et al. Stress incontinence injection therapy: what is best for our patients? Eur Urol 2005; 48: 552-565.

4. Corcos J, Fournier C. Periurethral collagen injection for the treatment of female stress urinary incontinence: 4-year follow-up results. Urology 1999; 54: 815-818.

5. Furuta A, Jankowski RJ, Pruchnic R, Yoshimura N, ChancelIor MB. The potential of muscle-derived stem cells for stress urinary incontinence. Expert Opin Biol Ther 2007; 7: 14831486.

6. Furuta A, Jankowski RJ, Honda M, Pruchnic R, Yoshimura $\mathrm{N}$, Chancellor MB. State of the art of where we are at using stem cells for stress urinary incontinence. Neurourol Urodyn 2007; 26: 966-971.

7. Kim YT, Kim DK, Jankowski RJ, Pruchnic R, Usiene I, de Miguel $F$, et al. Human muscle-derived cell injection in a rat model of stress urinary incontinence. Muscle Nerve 2007; 36: 391-393.

8. Carr LK, Steele D, Steele S, Wagner D, Pruchnic R, Jankowski RJ, et al. University of Toronto clinical trial of muscle-derived stem cell injection in women with stress urinary incontinence. J Urol 2007; 177: 439.

9. Muller-Ehmsen J, Whittaker P, Kloner RA, Dow JS, Sakoda $\mathrm{T}$, Long $\mathrm{TI}$, et al. Survival and development of neonatal rat cardiomyocytes transplanted into adult myocardium. $J \mathrm{Mol}$ Cell Cardiol 2002; 34: 107-116.

10. Reinecke H, Murry CE. Taking the death toll after cardiomyocyte grafting: a reminder of the importance of quantitative biology. J Mol Cell Cardiol 2002; 34: 251-253.

11. De Coppi P, Delo D, Farrugia L, Udompanyanan K, Yoo $\mathrm{JJ}$, Nomi M, et al. Angiogenic gene-modified muscle cells for enhancement of tissue formation. Tissue Eng 2005; 11:
1034-1044

12. Isner JM. Tissue responses to ischemia: local and remote responses for preserving perfusion of ischemic muscle. $J$ Clin Invest 2000; 106: 615-619.

13. Mann KG, Brummel $\mathrm{K}$, Butenas $\mathrm{S}$. What is all that thrombin for? J Thromb Haemost 2003; 1: 1504-1514.

14. Sarpel U, Roayaie S, Schwartz ME, Labow DM. The role of fibrin sealants in hepatic surgery. Surg Technol Int 2007; 16: 31-36.

15. Bootle-Wilbraham CA, Tazzyman S, Thompson WD, Stirk $\mathrm{CM}$, Lewis CE. Fibrin fragment $\mathrm{E}$ stimulates the proliferation, migration and differentiation of human microvascular endothelial cells in vitro. Angiogenesis 2001; 4: 269-275.

16. Herrick S, Blanc-Brude O, Gray A, Laurent G. Fibrinogen. Int J Biochem Cell Biol 1999; 31: 741-746.

17. Jockenhoevel S, Zund G, Hoerstrup SP, Chalabi K, Sachweh JS, Demircan L, et al. Fibrin gel - advantages of a new scaffold in cardiovascular tissue engineering. Eur J Cardiothorac Surg 2001; 19: 424-430.

18. Cummings CL, Gawlitta D, Nerem RM, Stegemann JP. Properties of engineered vascular constructs made from collagen, fibrin, and collagen-fibrin mixtures. Biomaterials 2004; 25: 3699-3706.

19. Hijaz A, Bena J, Daneshgari F. Long-term efficacy of a vaginal sling procedure in a rat model of stress urinary incontinence. J Urol 2005; 173: 1817-1819.

20. Qu-Petersen Z, Deasy B, Jankowski R, Ikezawa M, Cummins J, Pruchnic R, et al. Identification of a novel population of muscle stem cells in mice: potential for muscle regeneration. J Cell Biol 2002; 157: 851-864.

21. Lee JY, Qu-Petersen Z, Cao B, Kimura S, Jankowski R, Cummins $\mathrm{J}$, et al. Clonal isolation of muscle-derived cells capable of enhancing muscle regeneration and bone healing. J Cell Biol 2000; 150: 1085-1100.

22. Damaser MS, Broxton-King C, Ferguson C, Kim FJ, Kerns $\mathrm{JM}$. Functional and neuroanatomical effects of vaginal distention and pudendal nerve crush in the female rat. $J$ Urol 2003; 170: 1027-1031.

23. Cannon TW, Lee JY, Somogyi G, Pruchnic R, Smith CP, Huard J, et al. Improved sphincter contractility after allogenic 
muscle-derived progenitor cell injection into the denervated rat urethra. Urology 2003; 62: 958-963.

24. Liu Z, Wu Y, Chen BG. Myoblast therapy: from bench to bedside. Cell Transplant 2006; 15: 455-462.

25. Conway DA, Kamo I, Yoshimura N, Chancellor MB, Cannon TW. Comparison of leak point pressure methods in an animal model of stress urinary incontinence. Int Urogynecol J 2005; 16: 359-363.

26. Cannon TW, Damaser MS. Effects of anesthesia on cystometry and leak point pressure of the female rat. Life Sci 2001; 69: 1193-1202.

27. Ahn H, Lin DL, Esparza N, Damaser MS. Short-term timecourse of bilateral pudendal nerve injury on leak-point pressure in female rats. J Rehabil Res Dev 2005; 42: 109-114.

28. Chermansky CJ, Tarin T, Kwon DD, Jankowski RJ, Cannon TW, de Groat WC, et al. Intraurethral muscle-derived cell injections increase leak point pressure in a rat model of intrinsic sphincter deficiency. Urology 2004; 63: 780-785.

29. Furuta A, Jankowski RJ, Pruchnic R, Egawa S, Yoshimura $\mathrm{N}$, Chancellor MB. Physiological effects of human musclederived stem cell implantation on urethral smooth muscle function. Int Urogynecol J Pelvic Floor Dysfunct 2008; 19: 1229-1234.

30. Dedkov El, Kostrominova TY, Borisov AB, Carlson BM. Survival of Schwann cells in chronically denervated skeletal muscles. Acta Neuropathol 2002; 103: 565-574.

31. Ahmed TA, Dare EV, Hincke M. Fibrin: a versatile scaffold for tissue engineering applications. Tissue Eng Part B Rev 2008; 14: 199-215.

32. Ye Q, Zund G, Benedikt P, Jockenhoevel S, Hoerstrup SP, Sakyama $S$, et al. Fibrin gel as a three dimensional matrix in cardiovascular tissue engineering. Eur J Cardiothorac Surg 2000; 17: 587-591. 